\title{
SOME VERSIONS OF ANDERSON'S AND MAHER'S INEQUALITIES II
}

\section{SALAH MECHERI}

Received 11 January 2003

\begin{abstract}
We are interested in the investigation of the orthogonality (in the sense of Birkhoff) of the range of an elementary operator and its kernel.
\end{abstract}

2000 Mathematics Subject Classification: 47B47, 47A30, 47B10.

1. Introduction. Let $H$ be a separable infinite-dimensional complex Hilbert space and let $B(H)$ denote the algebra of all bounded operators on $H$ into itself. Given $A, B \in B(H)$, we define the generalized derivation $\delta_{A, B}: B(H) \mapsto B(H)$ by $\delta_{A, B}(X)=A X-X B$ and the elementary operator derivation $\Delta_{A, B}: B(H) \mapsto B(H)$ by $\Delta_{A, B}(X)=A X B-X$. Denote $\delta_{A, A}=\delta_{A}, \Delta_{A, A}=\Delta_{A}$.

In [1, Theorem 1.7], Anderson shows that if $A$ is normal and commutes with $T$, then, for all $X \in B(H)$,

$$
\left\|T+\delta_{A}(X)\right\| \geq\|T\|
$$

It is shown in [9] that if the pair $(A, B)$ has the Fuglede-Putnam property (in particular, if $A$ and $B$ are normal operators) and $A T=T B$, then, for all $X \in B(H)$,

$$
\left\|T+\delta_{A, B}(X)\right\| \geq\|T\|
$$

Duggal [3] showed that the above inequality (1.2) is also true when $\delta_{A, B}$ is replaced by $\Delta_{A, B}$. The related inequality (1.1) was obtained by the author [10], showing that if the pair $(A, B)$ has the Fuglede-Putnam property $(\mathrm{FP})_{C_{p}}$, then

$$
\left\|T+\delta_{A, B}(X)\right\|_{p} \geq\|T\|_{p}
$$

for all $X \in B(H)$, where $C_{p}$ is the von Neumann-Schatten class, $1 \leq p<\infty$, and $\|\cdot\|_{p}$ is its norm for all $X \in B(H)$ and for all $T \in C_{p} \cap \operatorname{ker} \delta_{A, B}$. In all of the above results, $A$ was not arbitrary. In fact, certain normality-like assumptions have been imposed on $A$. A characterization of $T \in C_{p}$ for $1<p<\infty$, which is orthogonal to $R\left(\delta_{A} \mid C_{p}\right)$ (the range of $\left.\delta_{A} \mid C_{p}\right)$ for a general operator $A$, has 
been carried out by Kittaneh [6], showing that if $T$ has the polar decomposition $T=U|T|$, then

$$
\left\|T+\delta_{A}(X)\right\|_{p} \geq\|T\|_{p}
$$

for all $X \in C_{p}(1<p<\infty)$ if and only if $|T|^{p-1} U^{*} \in \operatorname{ker} \delta_{A}$. By a simple modification in the proof of the above inequality, we can prove that this inequality is also true in the general case, that is, if $T$ has the polar decomposition $T=U|T|$, then

$$
\left\|T+\delta_{A, B}(X)\right\|_{p} \geq\|T\|_{p}
$$

for all $X \in C_{p}(1<p<\infty)$ if and only if $|T|^{p-1} U^{*} \in \operatorname{ker} \delta_{B, A}$. In Sections 1,2 , 3 , and 4 , we prove these results in the case where we consider $E_{A, B}$ instead of $\delta_{A, B}$, which leads us to prove that if $T \in C_{p}$ and $\operatorname{ker} E_{A, B} \subseteq \operatorname{ker} E_{A, B}^{*}$, then

$$
\left\|T+E_{A, B}(X)\right\|_{p} \geq\|T\|_{p}
$$

for all $X \in C_{p}(1<p<\infty)$ if and only if $T \in \operatorname{ker} E_{A, B}$. In Sections 5 , 6, and 7 , we minimize the map $\left\|S+E_{A, B}(X)\right\|_{p}$ and we classify its critical points.

2. Preliminaries. Let $T \in B(H)$ be compact and let $s_{1}(X) \geq s_{2}(X) \geq \cdots \geq 0$ denote the singular values of $T$, that is, the eigenvalues of $|T|=\left(T^{*} T\right)^{1 / 2}$ arranged in their decreasing order. The operator $T$ is said to belong to the Schatten $p$-class $C_{p}$ if

$$
\|T\|_{p}=\left[\sum_{i=1}^{\infty} s_{j}(T)^{p}\right]^{1 / p}=\left[\operatorname{tr}(T)^{p}\right]^{1 / p}, \quad 1 \leq p<\infty,
$$

where $\operatorname{tr}$ denotes the trace functional. Hence, $C_{1}$ is the trace class, $C_{2}$ is the Hilbert-Schmidt class, and $C_{\infty}$ is the class of compact operators with

$$
\|T\|_{\infty}=s_{1}(T)=\sup _{\|f\|=1}\|T f\|
$$

denoting the usual operator norm. For the general theory of the Schatten $p$ classes, the reader is referred to $[7,11]$.

Recall that the norm $\|\cdot\|$ of the $B$-space $V$ is said to be Gateaux differentiable at nonzero elements $x \in V$ if

$$
\lim _{t \rightarrow 0, t \in \mathbb{R}} \frac{\|x+t y\|-\|x\|}{t}=\mathscr{R} D_{x}(y)
$$

for all $y \in V$. Here $\mathbb{R}$ denotes the set of reals, $\mathscr{R}$ denotes the real part, and $D_{x}$ is the unique support functional (in the dual space $V^{*}$ ) such that $\left\|D_{x}\right\|=1$ and $D_{x}(x)=\|x\|$. The Gateaux differentiability of the norm at $x$ implies that $x$ is a smooth point of the sphere of radius $\|x\|$. 
It is well known (see [7] and the references therein) that, for $1<p<\infty, C_{p}$ is a uniformly convex Banach space. Therefore, every nonzero $T \in C_{p}$ is a smooth point and, in this case, the support functional of $T$ is given by

$$
D_{T}(X)=\operatorname{tr}\left[\frac{|T|^{p-1} U X^{*}}{\|T\|_{p}^{p-1}}\right]
$$

for all $X \in C_{p}$, where $T=U|T|$ is the polar decomposition of $T$.

DEFINITION 2.1. Let $E$ be a complex Banach space. We define the orthogonality in $E$. We say that $b \in E$ is orthogonal to $a \in E$ if, for all complex $\lambda$, there holds

$$
\|a+\lambda b\| \geq\|a\|
$$

This definition has a natural geometric interpretation, namely, $b \perp a$ if and only if the complex line $\{a+\lambda b \mid \lambda \in C\}$ is disjoint with the open ball $K(0,\|a\|)$, that is, if and only if this complex line is a tangent one. Note that if $b$ is orthogonal to $a$, then $a$ needs not be orthogonal to $b$. If $E$ is a Hilbert space, then from (2.5), it follows that $\langle a, b\rangle=0$, that is, orthogonality in the usual sense.

\section{The elementary operators $A X B-C X D$}

LEMMA 3.1. Let $A, B \in B(H)$. The following statements are equivalent:

(1) the pair $(A, B)$ has the property $(F P)_{C_{p}}, 1 \leq p<\infty$;

(2) if $A T=T B$, where $T \in C_{p}$, then $\overline{R(T)}$ reduces $A$, $\operatorname{ker}(T)^{\perp}$ reduces $B$, and $\left.A\right|_{\overline{R(T)}}$ and $\left.B\right|_{\operatorname{ker}(T) \perp}$ are normal operators.

Proof. $(1) \Rightarrow(2)$. Since $C_{p}$ is a bilateral ideal and $T \in C_{p}$, then $A T \in C_{p}$. Hence as $A T=T B$ and $(A, B)$ satisfies $(F P)_{C_{p}}, A^{*} T=T B^{*}$, and so, $\overline{R(T)}$ and $\operatorname{ker}(T)^{\perp}$ are reducing subspaces for $A$ and $B$, respectively. Since $A(A T)=$ $(A T) B$ implies that $A^{*}(A T)=(A T) B^{*}$ by $(F P)_{C_{p}}$ and the equality $A^{*} T=T B^{*}$ implies that $A^{*} A T=A A^{*} T$, thus we see that $\left.A\right|_{\overline{R(T)}}$ is normal. Clearly, $\left(B^{*}, A^{*}\right)$ satisfies $(F P)_{C_{p}}$ and $B^{*} T^{*}=T^{*} A^{*}$. Therefore, it follows from the above argument that $\left.B^{*}\right|_{R\left(T^{*}\right)}=\left.B\right|_{\operatorname{ker}(T)^{\perp}}$ is normal.

(2) $\Rightarrow(1)$. Let $T \in C_{p}$ such that $A T=T B$. Taking the two decompositions of $H, H_{1}=H=\overline{R(T)} \oplus \overline{R(T)}{ }^{\perp}$ and $H_{2}=H=\operatorname{ker}(T)^{\perp} \oplus \operatorname{ker} T$, then we can write $A$ and $B$ on $H_{1}$ into $H_{2}$, respectively:

$$
A=\left[\begin{array}{cc}
A_{1} & 0 \\
0 & A_{2}
\end{array}\right], \quad B=\left[\begin{array}{cc}
B_{1} & 0 \\
0 & B_{2}
\end{array}\right],
$$

where $A_{1}$ and $B_{1}$ are normal operators. Also we can write $T$ and $X$ on $H_{2}$ into $H_{1}$ :

$$
T=\left[\begin{array}{cc}
T_{1} & 0 \\
0 & 0
\end{array}\right], \quad X=\left[\begin{array}{ll}
X_{1} & X_{2} \\
X_{3} & X_{4}
\end{array}\right]
$$


It follows from $A T=T B$ that $A_{1} T_{1}=T_{1} B_{1}$. Since $A_{1}$ and $B_{1}$ are normal operators, then, by applying the Fuglede-Putnam theorem, we obtain $A_{1}^{*} T_{1}=T_{1} B_{1}^{*}$, that is, $A^{*} T=T B^{*}$.

THEOREM 3.2. Let $A, B \in B(H)$. If $A$ and $B$ are normal operators, then

$$
\|S-(A X-X B)\|_{p} \geq\|S\|_{p}
$$

for all $X \in C_{p}$ and for all $S \in \operatorname{ker} \delta_{A, B} \cap C_{p}(1 \leq p<\infty)$.

Proof. Let $S=U|S|$ be the polar decomposition of $S$, where $U$ is an isometry such that $\operatorname{ker} U=\operatorname{ker}|S|$. Since

$$
\left\|U^{*} S\right\|_{p} \leq\left\|U^{*}\right\|_{p}\|S\|_{p}=\|S\|_{p}
$$

for all $S \in C_{p}$, then

$$
\|S-(A X-X B)\|_{p}^{p} \geq\left\|U^{*}[S-(A X-X B)]||_{p}^{p}=\right\||S|-U^{*}(A X-X B) \|_{p}^{p},
$$

and we have

$$
|||S|-\left.U^{*}(A X-X B)\right|_{p} ^{p} \geq \sum_{n}\left|\left\langle\left[|S|-U^{*}(A X-X B)\right] \varphi_{n}, \varphi_{n}\right\rangle\right|^{p}
$$

for any orthonormal basis $\left\{\varphi_{n}\right\}_{n \geq 1}$ of $H$. Since $A S=S B$, and $A$ and $B$ are normal operators, it follows from the Fuglede-Putnam theorem that $S^{*} A=B S^{*}$. Consequently, $S^{*} A S=B S^{*} S$ or $S^{*} S B=B S^{*} S$, that is, $B|S|=|S| B$. Since $|S|$ is a compact normal operator and commutes with $B$, there exists an orthonormal basis $\left\{f_{k}\right\} \cup\left\{g_{m}\right\}$ of $H$ such that $\left\{f_{k}\right\}$ consists of common eigenvectors of $B$ and $|S|$, and $\left\{g_{m}\right\}$ is an orthonormal basis of ker $|S|$. Since $\left\{f_{k}\right\}$ is an orthonormal basis of the normal operator $B$, then there exists a scalar $\alpha_{k}$ such that $B f_{k}=\alpha_{k} f_{k}$ and $B^{*} f_{k}=\bar{\alpha}_{k} f_{k}$. Consequently,

$$
\begin{aligned}
\left\langle U^{*}(A X-X B) f_{k},|S| f_{k}\right\rangle & =\left\langle S^{*}(A X-X B) f_{k}, f_{k}\right\rangle \\
& =\left\langle\left(B\left(S^{*} X\right)-\left(S^{*} X\right) B\right) f_{k}, f_{k}\right\rangle=0,
\end{aligned}
$$

that is, $\left\langle U^{*}(A X-X B) f_{k}, f_{k}\right\rangle=0$.

In (3.6) take $\left\{\varphi_{n}\right\}=\left\{f_{k}\right\} \cup\left\{g_{m}\right\}$ as an orthonormal basis of $H$, then

$$
\begin{aligned}
\sum_{n} \mid & \left.\left\langle\left[|S|-U^{*}(A X-X B)\right] \varphi_{n}, \varphi_{n}\right\rangle\right|^{p} \\
& \geq \sum_{k}\left|\left\langle|S| f_{k}, f_{k}\right\rangle\right|^{p}+\sum_{m}\left|\left\langle U^{*}(A X-X B) g_{m}, g_{m}\right\rangle\right|^{p} \\
& \geq \sum_{k}\left|\left\langle|S| f_{k}, f_{k}\right\rangle\right|^{p}=\|S\|_{p}^{p} .
\end{aligned}
$$


LEMMA 3.3. Let $A, B \in B(H)$ satisfying $(F P)_{C_{p}}$. Then

$$
\|S+A X-X B\|_{p}^{p} \geq\|S\|_{p}^{p}
$$

for every operator $S \in \operatorname{ker} \delta_{A, B} \cap C_{p}(1<p<\infty)$ and for all $X \in C_{p}$.

Proof. If the pair $(A, B)$ satisfies the $(F P)_{C_{p}}$ property, then $\overline{R(S)}$ reduces $A$, $\operatorname{ker}^{\perp} S$ reduces $B$, and $\left.A\right|_{\overline{R(S)}}$ and $\left.B\right|_{\operatorname{ker}^{\perp} S}$ are normal operators. Letting $S_{0}$ : $\operatorname{ker}^{\perp} S \rightarrow \overline{R(S)}$ be the quasiaffinity defined by setting $S_{0} x=S x$ for each $x \in$ $\operatorname{ker}^{\perp} S$, it results that $\delta_{A_{1}, B_{1}}\left(S_{0}\right)=\delta_{A_{1}^{*}, B_{1}^{*}}\left(S_{0}\right)=0$. Let $A=A_{1} \oplus A_{2}$, with respect to $H=\overline{R(S)} \oplus \overline{R(S)}{ }^{\perp}, A=B_{1} \oplus B_{2}$, with respect to $H=\operatorname{ker}(S)^{\perp} \oplus \operatorname{ker} S$, and $X: \overline{R(S)} \oplus \overline{R(S)}{ }^{\perp} \rightarrow \operatorname{ker}(S)^{\perp} \oplus \operatorname{ker} S$ have the matrix representation

$$
X=\left[\begin{array}{ll}
X_{1} & X_{2} \\
X_{3} & X_{4}
\end{array}\right]
$$

Then we have

$$
\|S-(A X-X B)\|_{p}=\left\|\left[\begin{array}{cc}
S_{1}-\left(A_{1} X_{1}-X_{1} B_{1}\right) & * \\
* & *
\end{array}\right]\right\|_{p} .
$$

The result of Gohberg and Kreĭn [4] guarantees that

$$
\|S-(A X-X B)\|_{p} \geq\left\|S_{1}-\left(A_{1} X_{1}-X_{1} B_{1}\right)\right\|_{p} .
$$

Since $A_{1}$ and $B_{1}$ are two normal operators, then it results from Theorem 3.5 that

$$
\left\|S_{1}-\left(A_{1} X_{1}-X_{1} B_{1}\right)\right\|_{p} \geq\left\|S_{1}\right\|_{p}=\|S\|_{p} .
$$

LEMMA 3.4 [6]. Let $u$ and $v$ be two elements of a Banach space $V$ with norm $\|\cdot\|$. If $u$ is a smooth point, then $D_{u}(v)=0$ if and only if

$$
\|u+z v\| \geq\|u\|
$$

for all $z \in \mathbb{C}$ (the complex numbers).

THEOREM 3.5. Let $A, B \in B(H)$ and $T \in C_{p}(1<p<\infty)$. Then

$$
\left\|T+\delta_{A, B}(X)\right\|_{p} \geq\|T\|_{p}
$$

for all $X \in B(H)$ with $\Delta_{A, B}(X) \in C_{p}$ if and only if

$$
\operatorname{tr}\left(|T|^{p-1} U^{*} \delta_{A, B}(X)\right)=0
$$

for all such $X$. 
Proof. The theorem is an immediate consequence of equality (2.4) and Lemma 3.4.

THEOREM 3.6. Let $A, B \in B(H)$ and $T \in C_{p}(1<p<\infty)$. Then

$$
\left\|T+\delta_{A, B}(X)\right\|_{p} \geq\|T\|_{p}
$$

for all $X \in C_{p}$ if and only if $\tilde{T}=|T|^{p-1} U^{*} \in \operatorname{ker} \delta_{B, A}$.

Proof. By virtue of Theorem 3.5, it is sufficient to show that $\operatorname{tr}\left(\tilde{T} \delta_{A, B}(X)\right)=$ 0 for all $X \in C_{p}$ if and only if $\tilde{T} \in \operatorname{ker} \delta_{B, A}$.

Choose $X$ to be the rank-one operator $f \otimes g$ for some arbitrary elements $f$ and $g$ in $H$. Then $\operatorname{tr}(\tilde{T}(A X-X B))=\operatorname{tr}(B \tilde{T}-\tilde{T} A) X=0$ implies that $\left\langle\delta_{B, A}(\tilde{T}) f, g\right\rangle$ $=0 \Leftrightarrow \tilde{T} \in \operatorname{ker} \delta_{B, A}$.

Conversely, assume that $\tilde{T} \in \operatorname{ker} \delta_{B, A}$, that is, $B \tilde{T}=\tilde{T} A$. Since $\tilde{T} X$ and $\tilde{T} \delta_{B, A}$ are trace classes, then for all $X \in C_{p}$, we get

$$
\begin{aligned}
\operatorname{tr}(\tilde{T}(A X-X B)) & =\operatorname{tr}(\tilde{T} A X-\tilde{T} X B)=\operatorname{tr}(X B \tilde{T}-X \tilde{T} A) \\
& =\operatorname{tr}\left(X \delta_{B, A}(\tilde{T})\right)=0
\end{aligned}
$$

LEMMA 3.7. Let $A, B \in B(H)$ and $S \in C_{p}$ such that $\delta_{A, B}(T)=0=\delta_{A, B}^{*}(T)$.

If $A|S|^{p-1} U^{*}=|S|^{p-1} U^{*} B$, where $p>1$ and $S=U|S|$ is the polar decomposition of $S$, then $A|S| U^{*}=|S| U^{*} B$.

Proof. If $T=|S|^{p-1}$, then

$$
A T U^{*}=T U^{*} B
$$

We prove that

$$
A T^{n} U^{*}=T^{n} U^{*} B
$$

for all $n \geq 1$. If $S=U|S|$, then

$$
\begin{gathered}
\operatorname{ker} U=\operatorname{ker}|S|=\operatorname{ker}|S|^{p-1}=\operatorname{ker} T, \\
(\operatorname{ker} U)^{\perp}=(\operatorname{ker} T)^{\perp}=\overline{R(T) .}
\end{gathered}
$$

This shows that the projection $U^{*} U$ onto $(\operatorname{ker} T)^{\perp}$ satisfies $U^{*} U T=T$ and $T U^{*} U T=T^{2}$. By taking the adjoints of (3.19) and since $A$ and $B$ are normal operators applying Fuglede-Putnam theorem, we get $B U T=U T A$ and $A T^{2}=$ $A T U^{*} U T=T U^{*} B U T=T U^{*} U T A=T^{2} A$.

Since $A$ commutes with the positive operator $T^{2}, A$ commutes with its square roots, that is,

$$
A T=T A .
$$

By (3.19) and (3.22) we obtain (3.20). Let $f(t)$ be the map defined on $\sigma(T) \subset$ $\mathbb{R}^{+}$by $f(t)=t^{1 /(p-1)}(1<p<\infty)$. Since $f$ is the uniform limit of a sequence 
$\left(P_{i}\right)$ of polynomials without constant term (since $f(0)=0$ ), it follows from (3.20) that $A P_{i}(T) U^{*}=P_{i}(T) U^{*} B$. Therefore, $A T^{1 /(p-1)} U^{*}=U^{*} T^{1 /(p-1)} B$.

THEOREM 3.8. Let $A$ and $B$ be operators in $B(H)$ such that $\delta_{A, B}(T)=0=$ $\delta_{A, B}^{*}(T)$. Then $T \in \operatorname{ker} \Delta_{A, B} \cap C_{p}$ if and only if

$$
\left\|S+\delta_{A, B}(X)\right\|_{p} \geq\|S\|_{p}
$$

for all $X \in C_{p}$.

Proof. If $S \in \operatorname{ker} \Delta_{A, B}$, then it follows from Lemma 3.3 that

$$
\left\|S+\delta_{A, B}(X)\right\|_{p} \geq\|S\|_{p}
$$

for all $X \in C_{p}$. Conversely, if

$$
\left\|S+\delta_{A, B}(X)\right\|_{p} \geq\|S\|_{p}
$$

for all $X \in C_{p}$, then, from Theorem 3.6,

$$
A|S|^{p-1} U^{*}=|S|^{p-1} U^{*} B .
$$

Since $\delta_{A, B}(S)=0=\delta_{A, B} *(S)$,

$$
A^{*}|S|^{p-1} U^{*}=|S|^{p-1} U^{*} B^{*} .
$$

By taking adjoints, we get

$$
A U|S|^{p-1}=U|S|^{p-1} B .
$$

From Lemma 3.7, it follows that $A U|S|=U|S| B$, that is, $S \in \operatorname{ker} \Delta_{A, B}$.

REMARK 3.9. (1) It is well known that the Hilbert-Schmidt class $C_{2}$ is a Hilbert space under the inner product $\langle Y, Z\rangle=\operatorname{tr} Z^{*} Y$.

We remark here that for the Hilbert-Schmidt norm $\|\cdot\|_{2}$, the orthogonality result in Theorem 3.8 is to be understood in the usual Hilbert-space sense. Note in the case where $I=C_{2}$ that

$$
\left\|T+\delta_{A, B}(X)\right\|_{2}^{2}=\left\|\delta_{A, B}(X)\right\|_{2}^{2}+\|T\|_{2}^{2}
$$

for all $X \in C_{2}$ if and only if $A T^{*}=T^{*} B$. This can be seen as an immediate consequence of the fact that

$$
R\left(\delta_{A, B} \mid C_{2}\right)^{\perp}=\operatorname{ker}\left(\delta_{A, B} \mid C_{2}\right)^{*}=\operatorname{ker}\left(\delta_{B^{*}, A^{*}} \mid C_{2}\right) .
$$

(2) It is known [2] that if $A$ and $B$ are contractions and $S \in C_{p}$, then $\delta_{A^{*}, B^{*}}(S)$ $=\delta_{A, B}(S)=0$. Hence

$$
\left\|S+\delta_{A, B}(X)\right\|_{p} \geq\|S\|_{p}
$$

holds for all $X \in C_{p}$ if and only if $S \in \operatorname{ker}\left(\delta_{A, B} \mid C_{p}\right)$. 
(3) If $A=B$, then the following counterexample shows that Theorem 3.8 does not hold if $p<1$. Take $p=1 / 2$ and

$$
A=\left[\begin{array}{ll}
1 & 0 \\
0 & 0
\end{array}\right], \quad S=\left[\begin{array}{ll}
1 & 0 \\
0 & 1
\end{array}\right], \quad X=\left[\begin{array}{cc}
0 & -\alpha \\
\alpha & 0
\end{array}\right],
$$

where $\alpha$ is real such that $0<\alpha<1$. We have

$$
S-(A X-X A)=\left[\begin{array}{ll}
1 & \alpha \\
\alpha & 1
\end{array}\right]
$$

and, for eigenvectors $\beta_{1}=1-\alpha, \beta_{2}=1+\alpha$. Then

$$
\|S-(A X-X A)\|_{1 / 2}=\left[(1-\alpha)^{1 / 2}+(1+\alpha)^{1 / 2}\right]^{2}<4=\|S\|_{1 / 2} .
$$

COROLlary 3.10. Let $A, B \in L(H)$. Then

$$
\|S+A X-X B\|_{p} \geq\|S\|_{p}
$$

if and only if $S \in \operatorname{ker} \delta_{A, B} \cap C_{p}$ and for all $X \in C_{p}$, in each of the following cases:

(1) if $A, B \in L(H)$ such that $\|A x\| \geq\|x\| \geq\|B x\|$ for all $x \in H$,

(2) if $A$ is invertible and $B$ is such that $\left\|A^{-1}\right\|\|B\| \leq 1$.

Proof. The result of Tong [13, Lemma 1] guarantees that the above condition implies that, for all $T \in \operatorname{ker}\left(\delta_{A, B} \mid K(H)\right), \overline{R(T)}$ reduces $A$, $\operatorname{ker}(T)^{\perp}$ reduces $B$, and $\left.A\right|_{\overline{R(T)}}$ and $\left.B\right|_{\operatorname{ker}(T) \perp}$ are unitary operators. Hence it results from Lemma 3.1 that the pair $(A, B)$ has the property $(F P)_{K(H)}$ and the results hold by Theorem 3.8. Here $K(H)$ is the ideal of compact operators.

The above inequality holds in particular if $A=B$ is isometric; in other words, $\|A x\|=\|x\|$ for all $x \in H$.

(2) In this case, it suffices to take $A_{1}=\|B\|^{-1} A, B_{1}=\|B\|^{-1} B$.

Then $\left\|A_{1} x\right\| \geq\|x\| \geq\left\|B_{1} x\right\|$ and the result holds by (1) for all $x \in H$.

4. Orthogonality and the elementary operators $A X B-C X D$. Let $H$ be a separable infinite-dimensional complex Hilbert space and let $B(H)$ denote the algebra of all bounded operators on $H$ into itself. Given $A, B, C$, and $D$ normal operators in $B(H)$ such that $A C=C A, B D=D B$, we define the elementary operator $\Psi: B(H) \mapsto B(H)$ by $\Psi(X)=A X B-C X D$. We prove that if $T \in C_{p}$ $(1<p<\infty)$, then $\|T+\Phi(X)\|_{p} \geq\|T\|_{p}$ if and only if $T \in \operatorname{ker} \Phi$ for all $X \in C_{p}$.

By the same argument used in the proofs of Theorems 3.5 and 3.6, we prove the following theorems.

THEOREM 4.1. Let $A, B, C, D \in B(H)$ and $T \in C_{p}(1<p<\infty)$. Then

$$
\|T+\Psi(X)\|_{p} \geq\|T\|_{p}
$$


for all $X \in B(H)$ with $\Psi(X) \in C_{p}$ if and only if

$$
\operatorname{tr}\left(|T|^{p-1} U^{*} \Psi(X)\right)=0
$$

for all such $X$.

THEOREM 4.2. Let $A, B, C, D \in B(H)$ and $T \in C_{p}(1<p<\infty)$. Then

$$
\|T+\Psi(X)\|_{p} \geq\|T\|_{p}
$$

for all $X \in C_{p}$ if and only if $\tilde{T}=|T|^{p-1} U^{*} \in \operatorname{ker} \Psi$.

LEMMA 4.3. Let $A, B \in B(H)$ be normal operators and $A B=B A$. Suppose that $A S B=B S A, S \in C_{p}(1<p<\infty)$. If

$$
A U|S|^{p-1} B=B U|S|^{p-1} A,
$$

then

$$
A U|S| B=B U|S| A .
$$

Proof. Assume that $B^{-1} \in B(H)$. Then, from $A S B=B S A$ and $A B=B A$, we get $A B^{-1} S=S B^{-1} A$. Hence, applying the above lemma to the operators $A B^{-1}$, $B^{-1} A$, and $S$, we get

$$
A B^{-1} U|S|^{p-1}=U|S|^{p-1} B^{-1} A,
$$

which implies that

$$
A B^{-1} U|S|=U|S| B^{-1} A .
$$

Multiply (4.6) and (4.7) at right and left by $B$ to obtain

$$
B A B^{-1} U|S|^{p-1} B=B U|S|^{p-1} B^{-1} A B
$$

or

$$
A B B^{-1} U|S|^{p-1} B=B U|S|^{p-1} B^{-1} B A
$$

that is,

$$
A U|S|^{p-1} B=B U|S|^{p-1} A,
$$

which implies that

$$
A U|S| B=B U|S| A .
$$

Consider now the case when $B$ is injective, that is, $\operatorname{ker} B=\{0\}$. Let

$$
\Delta_{n}=\left\{\lambda \in \mathbb{C}:|\lambda| \leq \frac{1}{n}\right\}
$$

and let $E_{B}\left(\Delta_{n}\right)$ be the corresponding spectral projector. 
Putting

$$
P_{n}=I-E_{B}\left(\Delta_{n}\right),
$$

the subspace $P_{n} H$ reduces both operators $A$ and $B$ (since they commute and are normal). Hence, with respect to the decomposition

$$
\begin{gathered}
H=\left(I-P_{n}\right) H \oplus P_{n} H, \\
A=\left[\begin{array}{cc}
A_{1}^{(n)} & 0 \\
0 & A_{2}^{(n)}
\end{array}\right], \quad B=\left[\begin{array}{cc}
B_{1}^{(n)} & 0 \\
0 & B_{2}^{(n)}
\end{array}\right], \\
S=\left[\begin{array}{ll}
S_{11}(n) & S_{12}(n) \\
S_{21}(n) & S_{22}(n)
\end{array}\right], \quad X=\left[\begin{array}{ll}
X_{11}(n) & X_{12}(n) \\
X_{21}(n) & X_{22}(n)
\end{array}\right],
\end{gathered}
$$

it is easy to see that $B_{2}^{(n)}$ acting on $P_{n} H$ is invertible. Then, from $A S B=B S A$, it follows that

$$
A_{2}^{(n)} S_{22}(n) B_{2}^{(n)}=B_{2}^{(n)} S_{22}(n) A_{2}^{(n)}
$$

and, from $A B=B A$, we get $A_{2} B_{2}=B_{2} A_{2}$. Since

$$
A U|S|^{p-1} B=B U|S|^{p-1} A,
$$

according to the first part of the proof, it follows that

$$
A_{2}^{(n)} U\left|S_{22}(n)\right|^{p-1} B_{2}^{(n)}=B_{2}^{(n)} U\left|S_{22}(n)\right|^{p-1} A_{2}^{(n)},
$$

which implies that

$$
A_{2}^{(n)} U\left|S_{22}(n)\right| B_{2}^{(n)}=B_{2}^{(n)} U\left|S_{22}(n)\right| A_{2}^{(n)},
$$

so we have $A U|S| B=B U|S| A$. Assume now $\operatorname{ker} A \cap \operatorname{ker} B=\{0\}$.

Then $\operatorname{ker} B$ reduces $A$ and $P_{\operatorname{ker} B} A P_{\operatorname{ker} B}$ is injective. Let $H=\operatorname{ker} B \oplus H_{1}\left(H_{1}=\right.$ $H \ominus \operatorname{ker} B)$. Then we have

$$
A=\left[\begin{array}{cc}
A_{1} & 0 \\
0 & A_{2}
\end{array}\right], \quad B=\left[\begin{array}{cc}
0 & 0 \\
0 & B_{2}
\end{array}\right], \quad S=\left[\begin{array}{ll}
S_{11} & S_{12} \\
S_{21} & S_{22}
\end{array}\right],
$$

where $A_{1}, B_{2}$ are injective and their ranges are dense in subspaces they act on. We have

$$
A S B-B S A=\left[\begin{array}{cc}
0 & A_{1} S_{12} B_{2} \\
-B_{2} S_{21} A_{1} & A_{2} S_{22} B_{2}-B_{2} S_{22} A_{2}
\end{array}\right] .
$$


Now, if $A S B=B S A$, then $A_{2} S_{22} B_{2}=B_{2} S_{22} A_{2}, B_{2} S_{21} A_{1}=0$, and $A_{1} S_{12} B_{2}=0$, that is, $S_{21}=S_{12}=0$. It follows that

$$
S=\left[\begin{array}{cc}
S_{11} & 0 \\
0 & S_{22}
\end{array}\right]
$$

Since $A_{2} B_{2}=B_{2} A_{2}, A_{2} S_{22} B_{2}=B_{2} S_{22} A_{2}$, and $B_{2}$ is injective, and we have already proved that

$$
A_{2} U\left|S_{22}\right|^{p-1} B_{2}=B_{2} U\left|S_{22}\right|^{p-1} A_{2}
$$

implies

$$
A_{2} U\left|S_{22}\right| B_{2}=B_{2} U\left|S_{22}\right| A_{2},
$$

so we have $A U|S| B=B U|S| A$.

Let $\Phi(X)=A X B-B X A$. We prove the following theorem.

THEOREM 4.4. Let $A, B \in B(H)$ be normal operators, $A B=B A$, and $S \in C_{p}$ $(1<p<\infty)$. Then $S \in \operatorname{ker} \Phi$ if and only if

$$
\|S-(A X B-B X A)\|_{p} \geq\|S\|_{p}
$$

for all $X \in C_{p}$.

Proof. If $S \in \operatorname{ker} \Phi$, then, from [13, Theorem 3.4], it follows that

$$
\|S+\Phi(X)\|_{p} \geq\|S\|_{p}
$$

for all $X \in C_{p}$. Conversely, if

$$
\|S+\Phi(X)\|_{p} \geq\|S\|_{p}
$$

for all $X \in C_{p}$, then, from Theorem 4.2,

$$
A|S|^{p-1} U^{*} B=B|S|^{p-1} U^{*} A .
$$

Since $A$ and $B$ are normal operators applying Fuglede-Putnam theorem, we get $A^{*}|S|^{p-1} U^{*} B^{*}=B^{*}|S|^{p-1} U^{*} A^{*}$. By taking adjoints, we get $A U|S|^{p-1} B=$ $B U|S|^{p-1} A$.

From Lemma 4.3, it follows that $A U|S| B=B U|S| A$, that is, $S \in \operatorname{ker} \Phi$.

Let $\Psi(X)=A X B-C X D$.

THEOREM 4.5. Let $A, B, C, D \in B(H)$ be normal operators, $A C=C A, B D=$ $D B$, and $S \in C_{p}(1<p<\infty)$. Then $S \in \operatorname{ker} \Psi$ if and only if

$$
\|S-(A X B-C X D)\|_{p} \geq\|S\|_{p}
$$

for all $X \in C_{p}$. 
Proof. It suffices to take the Hilbert space $H \oplus H$ and the operators

$$
\begin{array}{rlrl}
A^{\sim} & =\left[\begin{array}{ll}
A & 0 \\
0 & D
\end{array}\right], & & B^{\sim}=\left[\begin{array}{ll}
C & 0 \\
0 & B
\end{array}\right], \\
S^{\sim} & =\left[\begin{array}{ll}
0 & S \\
0 & 0
\end{array}\right], & X^{\sim}=\left[\begin{array}{cc}
0 & X \\
0 & 0
\end{array}\right]
\end{array}
$$

and apply Theorem 4.4.

REMARK 4.6. The results of the above theorems can be obtained when the normality of $A$ and $B$ is replaced by some other condition, in particular, if $|A|=|B|,\left|A^{*}\right|=\left|B^{*}\right|$. In this case, it suffices to take

$$
\begin{aligned}
A^{\sim} & =\left[\begin{array}{cc}
0 & A^{*} \\
B & 0
\end{array}\right], & B^{\sim} & =\left[\begin{array}{cc}
0 & B^{*} \\
A & 0
\end{array}\right], \\
S^{\sim} & =\left[\begin{array}{ll}
0 & S \\
0 & 0
\end{array}\right], & X^{\sim} & =\left[\begin{array}{cc}
0 & X \\
0 & 0
\end{array}\right]
\end{aligned}
$$

and apply Lemma 4.3 and Theorem 4.4.

5. On minimizing $\|A X-X B-T\|_{p}^{p}$. Maher [8, Theorem 3.2] shows that if $A$ is normal and $S \in \operatorname{ker} \delta_{A} \cap C_{p}(1 \leq p<\infty)$, then the map $F_{p}$ defined by $F_{p}(X)=\|S-(A X-X A)\|_{p}^{p}$ has a global minimizer at $V$ if, and for $1<p<\infty$ only if, $A V-V A=0$.

In this section, we prove that if the pair $(A, B)$ has the property $(F P)_{C_{p}}$ (i.e., $A T=T B$, where $T \in C_{p}$, implies $\left.A^{*} T=T B^{*}\right), 1 \leq p<\infty$, and $S \in \operatorname{ker} \delta_{A, B} \cap C_{p}$, then the map $F_{p}$ defined by $F_{p}(X)=\|S-(A X-X B)\|_{p}^{p}$ has a global minimizer at $V$ if, and for $1<p<\infty$ only if, $A V-V B=0$. In other words, we have

$$
\|S-(A X-X B)\|_{p}^{p} \geq\|T\|_{p}^{p}
$$

if, and for $1<p<\infty$ only if, $A V-V B=0$. Thus in Halmos' terminology [5], the zero commutator is the commutator approximant in $C_{p}$ of $T$. Additionally, we show that if the pair $(A, B)$ has the property $(F P)_{C_{p}}$ and $S \in \operatorname{ker} \delta_{A, B} \cap C_{p}(1<$ $p<\infty)$, then the map $F_{p}$ has a critical point at $W$ if and only if $A W-W B=0$, that is, if $\mathscr{D}_{W} F_{p}$ is the Frechet derivative at $W$ of $F_{p}$, the set $\left\{W \in B(H): \mathscr{D}_{W} F_{p}=\right.$ 0 \} coincides with $\operatorname{ker} \delta_{A, B}$ (the kernel of $\delta_{A, B}$ ).

THEOREM 5.1 [9]. If $1<p<\infty$, then the map $F_{p}: C_{p} \mapsto \mathbb{R}^{+}$defined by $X \mapsto$ $\|X\|_{p}^{p}$ is differentiable at every $X \in C_{p}$ with derivative $\mathscr{D}_{X} F_{p}$ given by $\mathscr{D}_{X} F_{p}(T)=$ $p \operatorname{Re} \operatorname{tr}\left(|X|^{p-1} U^{*} T\right)$, where tr denotes trace, $\operatorname{Re} z$ is the real part of a complex number $z$, and $X=U|X|$ is the polar decomposition of $X$. If $\operatorname{dim} H<\infty$, then the same result holds for $0<p \leq 1$ at every invertible $X$.

THEOREM 5.2 [9]. If $U$ is a convex set of $C_{p}, 1<p<\infty$, then the map $X \mapsto$ $\|X\|_{p}^{p}$, where $X \in \mathcal{U}$, has at most a global minimizer. 
Definition 5.3. Let $u(A, B)=\left\{X \in B(H): A X-X B \in C_{p}\right\}$ and let $F_{p}: U_{\mapsto}$ $\mathbb{R}^{+}$be the map defined by $F_{p}(X)=\|T-(A X-X B)\|_{p}^{p}$, where $T \in \operatorname{ker} \delta_{A, B} \cap C_{p}$, $1 \leq p<\infty$.

6. Main results. By simple modifications in the proof of Lemma 3.7, we can prove the following lemma.

LEMmA 6.1. Let $A, B \in B(H)$ and $C \in B(H)$ such that the pair $(A, B)$ has the property $(F P)_{B(H)}$. If $A|S|^{p-1} U^{*}=|S|^{p-1} U^{*} B$, where $p>1$ and $S=U|S|$ is the polar decomposition of $S$, then $A|S| U^{*}=|S| U^{*} B$.

THEOREM 6.2. Let $A, B \in \mathscr{L}(H)$. If the pair $(A, B)$ has the property $(F P)_{C_{p}}$ and $S \in C_{p}$ such that $A S=S B$, then

(1) for $1 \leq p<\infty$, the map $F_{p}$ has a global minimizer at $W$ if, and for $1<$ $p<\infty$ only if, $A W-W B=0$;

(2) for $1<p<\infty$, the map $F_{p}$ has a critical point at $W$ if and only if $A W-$ $W B=0$;

(3) for $0<p \leq 1 \operatorname{dim} \mathscr{H}<\infty$ and $S-(A W-W B)$ is invertible, then $F_{p}$ has a critical point at $W$ if $A W-W B=0$.

Proof. Since the pair $(A, B)$ has the property $(F P)_{C_{p}}$, it follows from Lemma 3.3 that

$$
\|S-(A X-X B)\|_{p}^{p} \geq\|S\|_{p}^{p}
$$

that is, $F_{p}(X) \geq F_{p}(W)$.

Conversely, if $F_{p}$ has a minimum, then

$$
\|S-(A W-W B)\|_{p}^{p}=\|S\|_{p}^{p}
$$

Since $\mathcal{U}$ is convex, the set $\mathscr{V}=\{S-(A X-X B) ; X \in \mathcal{U}\}$ is also convex. Thus Theorem 5.2 implies that

$$
S-(A W-W B)=S .
$$

(2) Let $W, S \in U$ and let $\phi$ and $\varphi$ be two maps defined, respectively, by $\phi: X \mapsto S-(A X-X B)$ and $\varphi: X \mapsto\|X\|_{p}^{p}$.

Since the Frechet derivative of $F_{p}$ is given by

$$
\mathscr{D}_{W} F_{p}(T)=\lim _{h \rightarrow 0} \frac{F_{p}(W+h T)-F_{p}(W)}{h},
$$

it follows that

$$
\mathscr{D}_{W} F_{p}(T)=\left[\mathscr{D}_{S-(A W-W B)}\right](T B-A T) .
$$


If $W$ is a critical point of $F_{p}$, then $\mathscr{D}_{W} F_{p}(T)=0$ for all $T \in \mathcal{U}$. By applying Theorem 5.1, we get

$$
\begin{aligned}
\mathscr{D}_{W} F_{p}(T) & =p \operatorname{Retr}\left[|S-(A W-W B)|^{p-1} W^{*}(T B-A T)\right] \\
& =p \operatorname{Retr}[Y(T B-A T)]=0,
\end{aligned}
$$

where $S-(A W-W B)=W|S-(A W-W B)|$ is the polar decomposition of the operator $S-(A W-W B)$ and $Y=|S-(A W-W B)|^{p-1} W^{*}$. An easy calculation shows that $B Y-Y A=0$, that is,

$$
A|S-(A W-W B)|^{p-1} W^{*}=|S-(A W-W B)|^{p-1} W^{*} B .
$$

It follows from Lemma 6.1 that

$$
A|S-(A W-W B)| W^{*}=|S-(A W-W B)| W^{*} B .
$$

By taking adjoints and since the pair $(A, B)$ has the property $(F P)_{C_{p}}$, we get $A(T-(A W-W B))=(T-(A W-W B)) B$. Then $A(A W-W B)=(A W-W B) B$. Hence

$$
A W-W B \in R\left(\delta_{A, B}\right) \cap \operatorname{ker} \delta_{A, B}
$$

By the same argument used in the proof of Lemma 6.1 we can prove that

$$
\|S-(A X-X B)\| \geq\|S\|
$$

for all $X \in B(H)$ and for all $T \in B(H)$ and it results that $A W-W B=0$.

Conversely, if $A W=W B$, then $W$ is a minimum, and since $F_{p}$ is differentiable, then $W$ is a critical point.

(3) Suppose that $\operatorname{dim} H<\infty$. If $A W-W B=0$, then $S$ is invertible by hypothesis. Also $|S|$ is invertible, hence $|S|^{p-1}$ exists for $0<p \leq 1$ taking $Y=|S|^{p-1} U^{*}$, where $S=U|S|$ is the polar decomposition of $S$. Since $A S=S B$ implies that $S^{*} A=B S^{*}$, then $S^{*} A S=B S^{*} S$, and this implies that $|S|^{2} B=B|S|^{2}$ and $|S| B=$ $B|S|$.

Since $S^{*} A=B S^{*}$, that is, $|S| U^{*} A=B|S| U^{*}$, then $|S|\left(U^{*} A-B U^{*}\right)=0$, and since $B|S|^{p-1}=|S|^{p-1} B$, then

$$
B Y-Y A=B|S|^{p-1} U^{*}-|S|^{p-1} U^{*} A=|S|^{p-1}\left(B U^{*}-U^{*} A\right)
$$

so that $B Y-Y A=0$ and $\operatorname{tr}[(B Y-Y A) T]=0$ for every $T \in B(H)$. Since $S=$ $S-(A W-W B)$, then

$$
\begin{aligned}
0 & =\operatorname{tr}[Y T B-Y A T]=\operatorname{tr}[Y(T B-A T)] \\
& =p \operatorname{Retr}[Y(T B-A T)]=p \operatorname{Retr}\left[|S|^{p-1} U^{*}(T B-A T)\right] \\
& =\left(\mathscr{D}_{T} \phi\right)(T B-A T)=\left(\mathscr{D}_{W} F_{p}\right)(T) .
\end{aligned}
$$


REMARK 6.3. In Theorem 6.2 , the implication " $W$ is a critical point implies $A W-W B=0$ " does not hold in the case $0<p \leq 1$ because the functional calculus argument involving the function $t \mapsto t^{1 /(p-1)}$, where $0 \leq t<\infty$, is only valid for $1<p<\infty$.

7. On minimizing $\|T-(A X B-C X D)\|_{p}^{p}$. In this section, we consider the elementary operator $\Phi(X)=A X B-C X D$ and we prove that if $A C=C A, B D=$ $D B$, and $A S B=C S D, S \in C_{p}$, then, for $1<p<\infty$, the map $F_{p}$ defined by $F_{p}(X)=\|T-(A X B-C X D)\|_{p}^{p}$ has a global minimizer at $V$ if, and for $1<p<\infty$ only if, $A V B-C V D=0$. In other words, we have $\|T-(A X B-C X D)\|_{p}^{p} \geq\|T\|_{p}^{p}$ if, and for $1<p<\infty$ only if, $A V B-C V D=0$. Additionally, we show that if $A C=C A, B D=D B$, and $T \in \operatorname{ker} \Delta_{A, B} \cap C_{p}, 1<p<\infty$, then the map $F_{p}$ has a critical point at $W$ if and only if $A W B-C W D=0$, that is, if $\mathscr{D}_{W} F_{p}$ is the Frechet derivative at $W$ of $F_{p}$, the set $\left\{W \in B(H): \mathscr{D}_{W} F_{p}=0\right\}$ coincides with $\operatorname{ker} \Phi$ (the kernel of $\Phi)$.

Definition 7.1. Let $u(A, B)=\left\{X \in B(H): A X B-C X D \in C_{p}\right\}$ and let $F_{p}$ : $\mathcal{U} \mapsto \mathbb{R}^{+}$be the map defined by $F_{p}(X)=\|T-(A X B-C X D)\|_{p}^{p}$, where $T \in$ $\operatorname{ker} \Phi \cap C_{p}, 1 \leq p<\infty$.

The proof of the following lemma is similar to the proof of Lemma 4.3.

LEMMA 7.2. Let $A, B \in B(H)$ be normal commuting operators. Suppose that $A S B=B S A, S \in C_{p}(1<p<\infty)$. If

$$
A|S|^{p-1} U^{*} B=B|S|^{p-1} U^{*} A,
$$

then

$$
A|S| U^{*} B=B|S| U^{*} A .
$$

THEOREM 7.3. Let $A, B, C, D \in B(H)$ be normal operators such that $A C=C A$ and $B D=D B$. Assume that $A S B=C S D, S \in C_{p}(1<p<\infty)$. If $A|S|^{p-1} U^{*} B=$ $C|S|^{p-1} U^{*} D$, then $A|S| U^{*} B=C|S| U^{*} D$.

Proof. It suffices to take the Hilbert space $H \oplus H$ and the operators

$$
A^{\sim}=\left[\begin{array}{cc}
A & 0 \\
0 & D
\end{array}\right], \quad B^{\sim}=\left[\begin{array}{ll}
C & 0 \\
0 & B
\end{array}\right], \quad S^{\sim}=\left[\begin{array}{ll}
0 & S \\
0 & 0
\end{array}\right]
$$

and apply Lemma 7.2.

THEOREM 7.4. Let $A, B, C, D \in B(H)$ be normal operators, $A C=C A$, and $B D=D B$. Suppose that $A S B=C S D, S \in C_{p}$. Then, for $1 \leq p<\infty$, the map $F_{p}$ has a global minimizer at $W$ if, and for $1<p<\infty$ only if, $A W B-C W D=0$. 
Proof. If $A C=C A, B D=D B$, and $A S B=C S D, S \in C_{p}$, then, for $1<p<\infty$, the result of Turnšek [14, Theorem 3.4] guarantees that

$$
\|T-(A X B-C X D)\|_{p}^{p} \geq\|T\|_{p}^{p},
$$

that is, $F_{p}(X) \geq F_{p}(W)$. Conversely, if $F_{p}$ has a minimum, then

$$
\|T-(A W B-C W D)\|_{p}^{p}=\|S\|_{p}^{p} .
$$

Since $\mathcal{U}$ is convex, then the set $\mathscr{V}=\{T-(A X B-C X D) ; X \in \mathcal{U}\}$ is also convex. Thus Theorem 5.2 implies that $S-(A W B-C W D)=S$.

THEOREM 7.5. Let $A, B, C$, and $D$ be normal operators in $B(H)$ such that $A C=C A$ and $B D=D B$. If $S \in \operatorname{ker} \Phi \cap C_{p}$, then, for $1<p<\infty$, the map $F_{p}$ has a critical point at $W$ if and only if $A W B-C W D=0$.

Proof. Let $W, S \in \mathcal{U}$ and let $\phi$ and $\varphi$ be two maps defined, respectively, by $\phi: X \mapsto S-(A X B-C X D)$ and $\varphi: X \mapsto\|X\|_{p}^{p}$. Since the Frechet derivative of $F_{p}$ is given by

$$
\mathscr{D}_{W} F_{p}(T)=\lim _{h \rightarrow 0} \frac{F_{p}(W+h T)-F_{p}(W)}{h},
$$

it follows that $\mathscr{D}_{W} F_{p}(T)=\left[\mathscr{D}_{S-(A W B-C W D)}\right](B T A-D T C)$. If $W$ is a critical point of $F_{p}$, then $\mathscr{D}_{W} F_{p}(T)=0$ for all $T \in \mathcal{U}$. By applying Theorem 5.1, we get

$$
\begin{aligned}
\mathscr{D}_{W} F_{p}(T) & =p \operatorname{Retr}\left[|S-(A W B-C W D)|{ }^{p-1} W^{*}(B T A-D T C)\right] \\
& =p \operatorname{Retr}[Y(B T A-D T C)]=0,
\end{aligned}
$$

where $S-(A W B-C W D)=W|S-(A W B-C W D)|$ is the polar decomposition of the operator $S-(A W B-C W D)$ and $Y=|S-(A W B-C W D)|^{p-1} W^{*}$. An easy calculation shows that $B Y A-D Y C=0$, that is,

$$
A|S-(A W B-C W D)|^{p-1} W^{*} B=C|S-(A W B-C W D)|^{p-1} W^{*} D .
$$

It follows from Theorem 7.3 that

$$
A|S-(A W B-C W D)| W^{*} B=C|S-(A W B-C W D)| W^{*} D .
$$

By taking adjoints and since $A$ and $B$ are normal operators, applying FugledePutnam theorem, we get $A(T-(A W B-C W D)) B=C(T-(A W B-C W D)) D$. Then $A(A W-W B) B=C(A W B-C W D) D$. Hence $A W B-C W D \in R(\Phi) \cap \operatorname{ker} \Phi$. By the same argument used in the proof of [13, Theorem 3.4], we can prove that

$$
\|T-(A X B-C X D)\| \geq\|T\|
$$

for all $T \in B(H)$. Hence $A W B-C W D=0$. 
Conversely, if $A W B=C W D$, then $W$ is a minimum, and since $F_{p}$ is differentiable, then $W$ is a critical point.

THEOREM 7.6. Let $A, B, C$, and $D$ be normal operators in $B(H)$ such that $A C=C A$ and $B D=D B$. If $S \in \operatorname{ker} \Phi \cap C_{p}, 0<p \leq 1, \operatorname{dim} H<\infty$, and $S-(A W B-$ $C W D)$ is invertible, then $F_{p}$ has a critical point at $W$ if $A W B-C W D=0$.

Proof. Suppose that $\operatorname{dim} H<\infty$. If $A W B-C W D=0$, then $S$ is invertible by hypothesis. Also $|S|$ is invertible, hence $|S|^{p-1}$ exists for $0<p \leq 1$. Taking $Y=|S|^{p-1} U^{*}$, where $S=U|S|$ is the polar decomposition of $S$, choose $X$ to be the rank-one operator $f \otimes g$ for some arbitrary elements $f$ and $g$ in $H \oplus H$. Then $\operatorname{tr}(Y(A X B-C X D))=\operatorname{tr}(A Y B-C Y D) X=0$ implies that $\langle\Psi(Y) f, g\rangle=0 \Leftrightarrow Y \in$ $\operatorname{ker} \Phi$, that is, $A Y B-C Y D=0$ and $\operatorname{tr}[(D Y C-A Y B) T]=0$ for every $T \in B(H)$. Since $S=S-(A W B-C W D)$, then

$$
\begin{aligned}
0 & =\operatorname{tr}[Y D T C-Y A T B]=\operatorname{tr}[Y(D T C-A T B)] \\
& =p \operatorname{Re} \operatorname{tr}[Y(D T C-A T B)]=p \operatorname{Retr}\left[|S|^{p-1} U^{*}(D T C-A T B)\right] \\
& =\left(\mathscr{D}_{T} \phi\right)(D T C-A T B)=\left(\mathscr{D}_{W} F_{p}\right)(T) .
\end{aligned}
$$

REMARK 7.7. The set $\mathscr{S}=\left\{X: A X B-C X D \in C_{p}\right\}$ contains $C_{p}$; if $X \in C_{p}$, then $X \in \mathscr{Y}$ and, for example, $I \in \mathscr{Y}$ but $I \notin C_{p}$. If $A \in C_{p}$, the conclusions of Theorems 7.3, 7.4, 7.5, and 7.6 hold for all $X \in B(H)$.

For $n>2$ the generalization of the above results to the elementary operators $\sum_{i=1}^{n} A_{i} X B_{i}$ is not possible. In [12], Shul'man stated that there exists a normally represented elementary operator of the form $\sum_{i=1}^{n} A_{i} X B_{i}$ with $n>2$ such that $\operatorname{asc} E>1$, that is, the range and kernel have no trivial intersection.

ACKNOWLEdgment. This work was supported by the Research Center Project no. Math/1422/10.

\section{REFERENCES}

[1] J. H. Anderson, On normal derivations, Proc. Amer. Math. Soc. 38 (1973), 135140.

[2] B. P. Duggal, On intertwining operators, Monatsh. Math. 106 (1988), no. 2, 139148.

[3] _ A remark on normal derivations, Proc. Amer. Math. Soc. 126 (1998), no. 7, 2047-2052.

[4] I. C. Gohberg and M. G. Krein, Introduction to the Theory of Linear Nonselfadjoint Operators, Translations of Mathematical Monographs, vol. 18, American Mathematical Society, Rhode Island, 1969.

[5] P. R. Halmos, Positive approximants of operators, Indiana Univ. Math. J. 21 (1971/1972), 951-960.

[6] F. Kittaneh, Operators that are orthogonal to the range of a derivation, J. Math. Anal. Appl. 203 (1996), no. 3, 868-873.

[7] F. Kittaneh and R. Younis, Smooth points of certain operator spaces, Integral Equations Operator Theory 13 (1990), no. 6, 849-855. 
[8] P. J. Maher, Commutator approximants, Proc. Amer. Math. Soc. 115 (1992), no. 4, 995-1000.

[9] S. Mecheri, On minimizing $\|S-(A X-X B)\|_{p}^{p}$, Serdica Math. J. 26 (2000), no. 2, 119-126.

[10] - On the orthogonality in von Neumann-Schatten class, Int. J. Appl. Math. 8 (2002), no. 4, 441-447.

[11] R. Schatten, Norm Ideals of Completely Continuous Operators, Ergebnisse der Mathematik und ihrer Grenzgebiete. N. F., vol. 27, Springer-Verlag, Berlin, 1960.

[12] V. S. Shul'man, Linear equations with normal coefficients, Dokl. Akad. Nauk SSSR 270 (1983), no. 5, 1070-1073 (Russian).

[13] Y. S. Tong, Kernels of generalized derivations, Acta Sci. Math. (Szeged) 54 (1990), no. 1-2, 159-169.

[14] A. Turnšek, Orthogonality in $\mathfrak{b}_{p}$ classes, Monatsh. Math. 132 (2001), no. 4, 349354.

Salah Mecheri: Department of Mathematics, College of Science, King Saud University, P.O. Box 2455, Riyadh 11451, Saudi Arabia

E-mail address: mecheri@ksu. edu.sa 


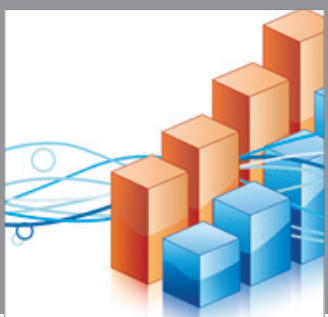

Advances in

Operations Research

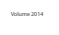

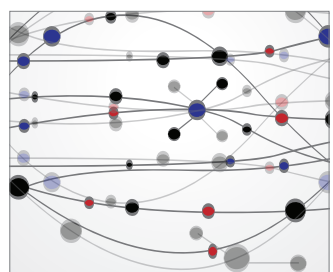

\section{The Scientific} World Journal
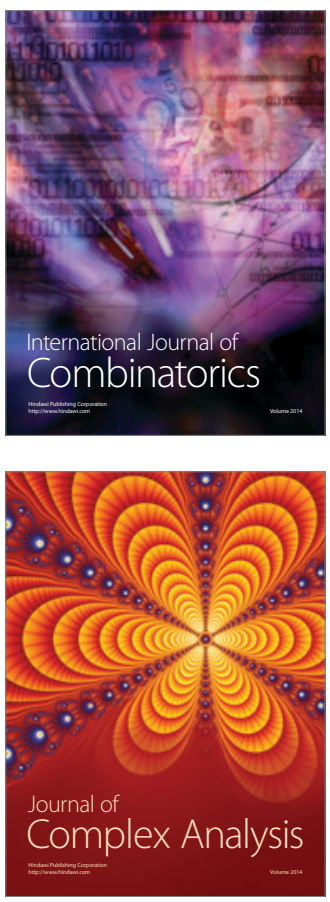

International Journal of

Mathematics and

Mathematical

Sciences
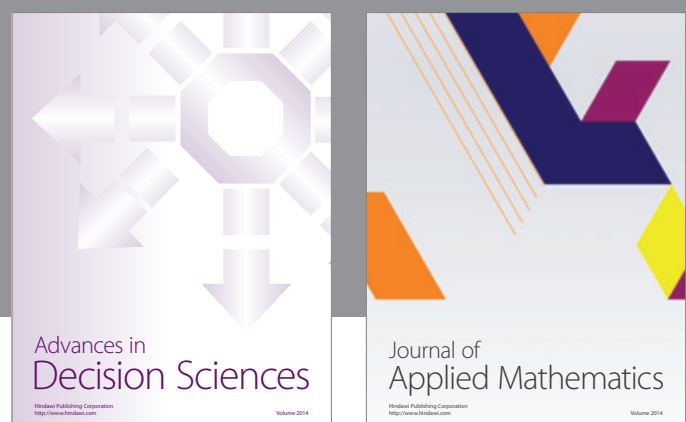

Journal of

Applied Mathematics
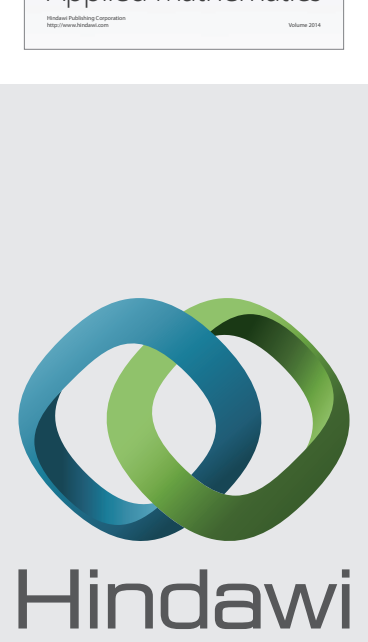

Submit your manuscripts at http://www.hindawi.com
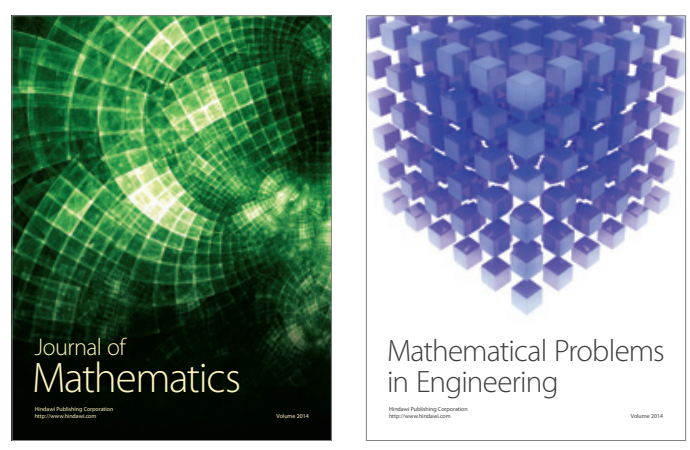

Mathematical Problems in Engineering
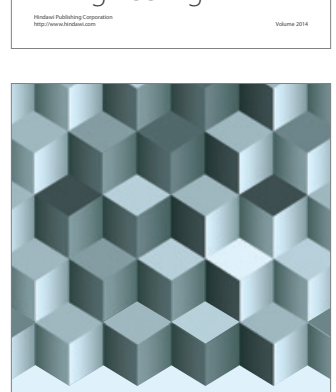

Journal of

Function Spaces
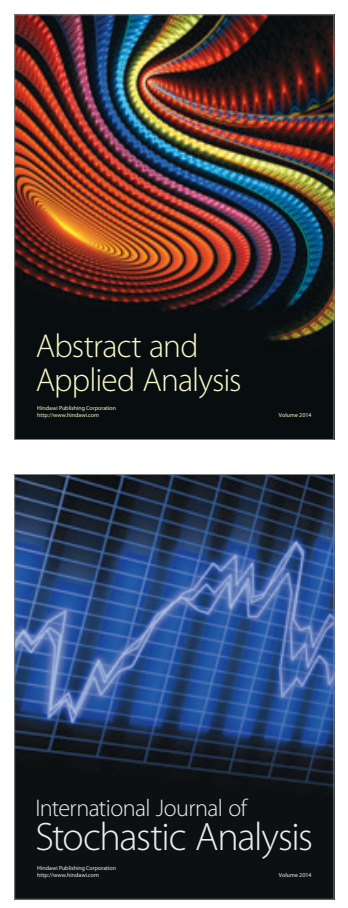

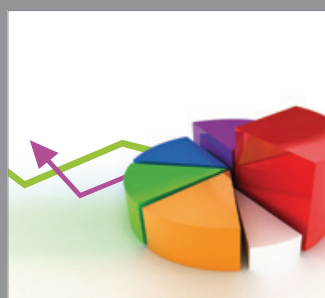

ournal of

Probability and Statistics

Promensencen
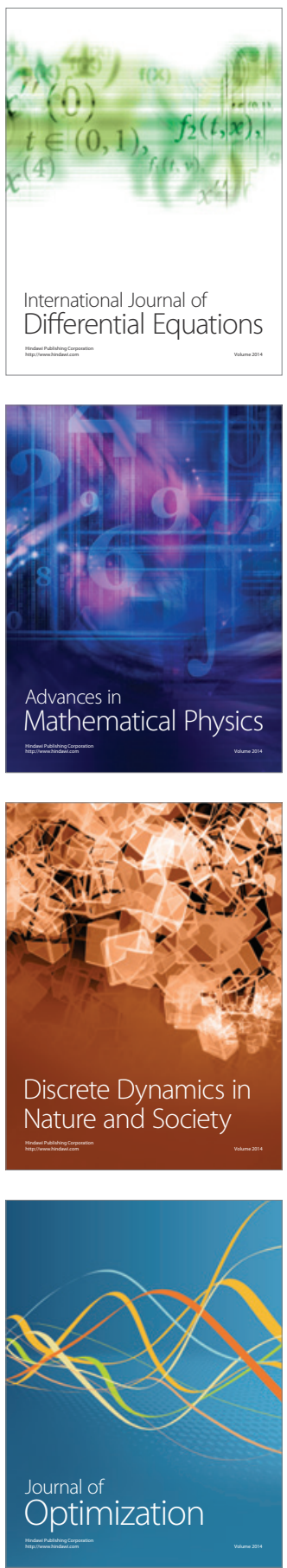